\title{
Evaluation of ECGI Localization Accuracy for Single Pacings in CRT Patients
}

\author{
Danila Potyagaylo $^{1}$, Mikhail Chmelevsky ${ }^{1,2}$, Stepan Zubarev ${ }^{2}$, Margarita Budanova ${ }^{2}$, Vitaly Kalinin ${ }^{1}$, \\ Alexander Kalinin ${ }^{1}$, Dmitry Lebedev ${ }^{2}$ \\ ${ }^{1}$ EP Solutions SA, Yverdon-les-Bains, Switzerland \\ ${ }^{2}$ Almazov National Medical Research Centre, Saint Petersburg, Russia
}

\begin{abstract}
ECG imaging (ECGI) is a noninvasive tool for estimating cardiac electrical activity from body surface potentials. Recently, there have been significant methodological improvements as well as a tremendous progress in the validation of ECGI reflected by an increasing number of published studies. Notwithstanding this progress, ECGI has neither been integrated into the clinical workflow concept nor achieved a clear positioning regarding its practical benefits involving cost and procedural time reduction. One of the main hurdles lies in the scarcity of high quality validation data. With this respect, the goal of the present study is to evaluate performance of three well-established inverse methods for ten cardiac resynchronization therapy (CRT) patients: the model-based fastest route algorithm (FRA), data-driven Tikhonov regularization, and a FRAbased maximum a posteriori methods were analyzed. Locations of the implanted pacing leads were exactly known from CT images, making them perfectly suited for ECGI accuracy assessment of focal activation sequences.
\end{abstract}

\section{Introduction}

ECG imaging (ECGI) is a noninvasive tool for estimating cardiac electrical activity from body surface potential maps (BSPMs). Based on the dipole cardiac electrical source model from 1962 [1], first attempts of solving the inverse problem of ECG can be traced back to 1972 [2]. In [3], Barr and Spach formulated the inverse problem in terms of epicardial potentials, conveying more information and exhibiting advantageous interpretability compared to the dipole current generator. Multifaceted benefits delivered by noninvasive imaging of extracellular cardiac potentials were shown in the pioneering works of Rudy et al. $[4,5]$, who developed the ECGI methodology from purely analytical studies to clinical applications [6].

Further source formulations include volumetric transmembrane potentials (TMPs) [7,8], which is the model closest to the actual cardiac generator, and their conditional surface counterpart. Assuming equal anisotropy ratios in extra- and intracellular domains, the cardiac electrical activity can be uniquely expressed by the TMPs on endo- and epicardial surfaces [9]. The equivalent dipole layer (EDL) model is an alternative formulation of the surface TMPs describing the cardiac excitation in terms of a dipole layer with normal orientation relative to the heart surface and strength proportional to TMP values [10]. It is closely related to the activation/recovery source depiction, where the temporal course of a TMP can effectively be characterized by two parameters, i.e. depolarization time and activationrecovery interval [11]. When imaging the excitation sequence, only the first parameter is of interest. Although this results in a still severely ill-posed problem, the inverse procedure can be strongly facilitated by incorporating a realistic propagation model [12].

Along methodological improvements, there has also been a tremendous progress in the validation of ECGI reflected by an increasing number of published studies. Having started from in silico investigations, torso tank experiments, and in vivo animal studies, ECGI reached the maturity state of being applied to humans for pathology mechanisms' understanding [6]. Furthermore, it has become increasingly popular as an academic research tool, whilst also enjoying a keen interest from the clinical community. The translational research profits from several commercially available ECGI systems working on proving their daily-use benefits (see e.g. Ref. [13]).

Despite this progress, ECGI has neither been integrated into the clinical workflow concept nor achieved a clear positioning regarding its practical benefits involving cost and procedural time reduction. One of the main hurdles lies in the scarcity of high quality validation data. Other obstacles as well as difficulties associated with designing ECGI validation studies are discussed in depth in Ref. [6].

With this respect, the goal of the present study is to evaluate performance of three well-established inverse methods for ten cardiac resynchronization therapy (CRT) patients. Locations of the implanted pacing leads were exactly known from CT images, making them perfectly suited for ECGI accuracy assessment of focal activation sequences. 


\section{Methods}

\subsection{Clinical data}

Ten patients with previously implanted CRT devices were subject to right- and leftventricular isolated pacings and subsequent noninvasive imaging. Consequently, we analyzed twenty paced BSPMs in total. All patients exhibited a low conduction velocity (CV) region in the left ventricle (LV). Note that six of them have had a myocardial infarction there.

We used a 240-channel BSPM Amycard 01C EP system ECG (EP Solutions SA, Switzerland) for electrocardiogram recording. The implanted CRT device was reprogrammed for isolated RV and LV pacings from implanted leads for $10 \mathrm{sec}$, after which the original parameter settings were restored. The absence of fusion beats served as criterion for an effective pacing capture. Directly after the BSPM measurements, all patients underwent CT imaging with applied electrode stripes for their precise localization. We employed the Amycard 01C EP system software for processing the obtained CT data and reconstruction of polygonal torso, lung and ventricular heart models.

The study was approved by the local institutional ethical board. Written informed consent was obtained from each patient after a detailed description and explanation of the study goals.

\subsection{Inverse reconstructions}

For the inverse modeling, we applied three previously published methods: Tikhonov regularization combined with a low-order parameterizaiton of BSPMs [14], fastest route algorithm (FRA) [12], and maximum a posteriori (MAP) approach [15] with the basis constructed by the FRA sequences. For all methods, we used the surface TMPs as a source model and a boundary element method (BEM) for relating them to the body surface ECGs, i.e. calculating the forward matrix. This transfer matrix is fully characterized by the volume conductor model and electrode positions.

Within the first methodology, BSPM signals were first projected onto a lower dimensional manifold using a spline-based approximation. Then, we applied classical Tikhonov regularization to the spline knot points, whose locations on the BSPM curves were found by nonlinear fitting. Finally, the obtained solution was interpolated from the abstract low-dimensional space back to the actual heart potentials [14]. As a regularization matrix, we utilized a single layer BEM matrix on the heart surface for taking into account transmural connections between epi- and endocardial surfaces.

FRA is a model-based full-search procedure, that was reported to be especially efficient for noninvasive imag- ing of focal arrhythmias $[12,13]$. Guided by a simple but physiologically meaningful shortest path excitation model, FRA treats each cardiac node as an activation origin and computes the corresponding propagation sequence on the heart. By means of the transfer matrix, these test sequences are linked to the respective BSPMs, whose durations are scaled to match the observed ECG signals. Then, the correlation coefficient (CC) is calculated between each test and reference (measured) BSPMs. The node exhibiting the highest correlation is taken as a solution approximation, whereby the resulting CC map can be visualized on the heart surface for an uncertainty estimation. Furthermore, the associated propagation sequence can be used as an initial guess for subsequent nonlinear least-squares (NNLS) optimization to find the activation times [12].

The aforementioned CC maps convey, in a sense, a probabilistic information on plausibility of FRA-propagated activation sequences. Therefore, these excitation patterns could be employed in any model-based inverse procedure. In order to reduce the computational load as well as further restrict the solution basis, only the sequences resulting in a high correlation can be taken into further considerations. In this work, we used the MAP approach with the source space constructed by the FRA excitations delivering a CC greater than 0.5 . As the activation sequences were scaled to match the measured BSPM duration, we did not restrict the solution interval by only an onset time window, as e.g. in Ref [16], and treated the whole QRS complex for inverse reconstructions.

For spline-based Tikhonov and MAP methods, we used two constant regularization parameters for $\mathrm{LV}$ and $\mathrm{RV}$ in all patients. Detection of earliest excitation sites was realized by taking the cardiac nodes, whose TMPs exceeded the $30 \%$ threshold of the overall potential value, and returning their geometric center of mass.

\subsection{Quality metrics}

In this study, ECGI could be validated solely against exact pacemaker positions, qualifying the localization error distances as a quality metric. As the LV implanted leads were situated on the epicardium and the RV ones on endocardium and septum, we also analyzed whether activation origins were inversely correctly localized on the the same (epi- or endocardial) heart surface.

Furthermore, we estimated the earliest excitation sites on epi- and endocardium for each inverse solution. For these points, we calculated either a geodesic or euclidean distance to the pacemaker, depending on its actual position. Geodesic distances are more representative for reconstructions on the curved LV surface and different sides of the endocardial septum and were computed for the onset situated on the 'pacemaker surface': epicardium in the LV or endocardium in the RV. The geodesic errors 

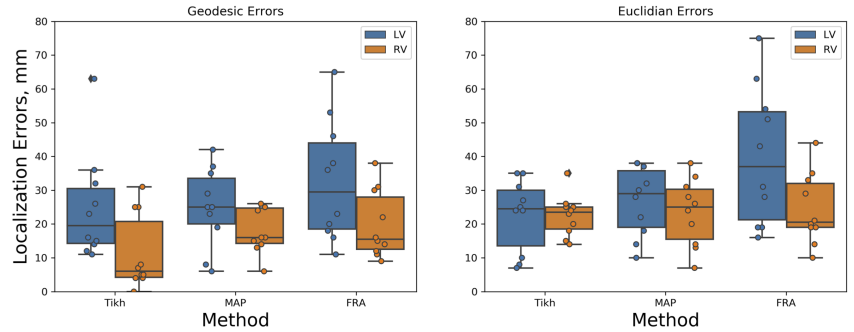

Figure 1: Boxplots for ECGI localization accuracy. Similar geodesic and euclidean distance errors reflect the methods' ability to noninvasively image both epi- and endocardial heart surfaces.

were used to estimate the intrinsic ill-posedness of the epi/endocardial inverse ECG problem, which needs either different amount of regualarization for each part of the heart surface or a special type of regularization matrices [14].

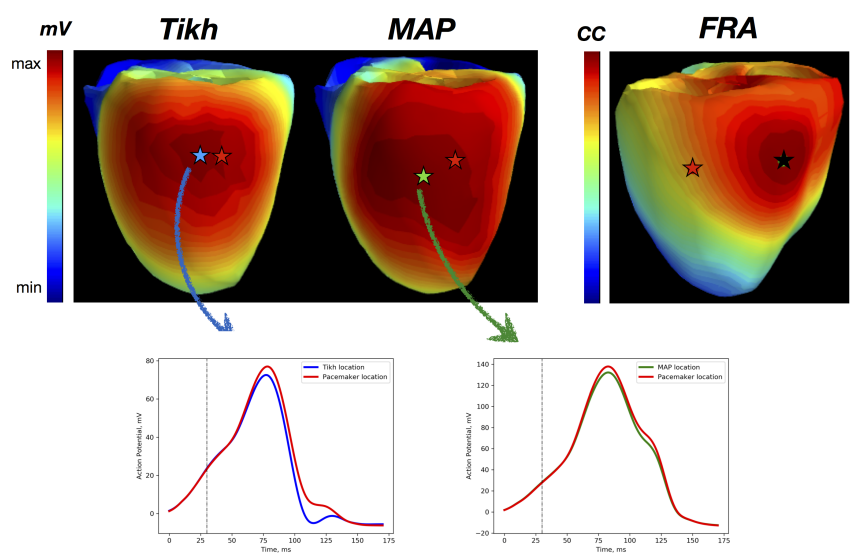

Figure 2: Exemplary reconstruction results for an epicardial LV pacing marked by a red star. Upper row: For Tikhonov and MAP methods, TMP distributions are visualized for the time step corresponding to a dashed vertical line on the plots below. The noninvasively localized origins are highlighted by blue and green stars, respectively. On the CC map delivered by FRA, the node exhibiting the highest correlation is indicated by a black star. Bottom row: Time course of reconstructed TMPs. The red line corresponds to the node closest to the actual pacing, while blue and green lines represent the origins localized by Tikhonov and MAP approaches, respectively.

\section{Results}

As displayed in Fig. 1, the reconstruction accuracy in the RV was higher than in the LV. Furthermore, all methods provided very similar localization estimates for the RV, while the LV cases featured increased FRA-related error distances. Exemplary instantaneous TMP maps for Tikhonov and MAP methods as well as FRA-based CC maps are visualized in Fig. 2,3. For Tikhonov and MAP methods, areas with the highest TMP values were smoothed out, hindering an accurate estimation of the earliest excitation site.

For evaluation of the ECGI method's ability to distinguish between endo- and epicardial sources, we provide the corresponding countplots in Fig. 4. While LV pacings were associated to an epicardial origin in most of the cases, the RV reconstructions demonstrated a very poor classification rate.

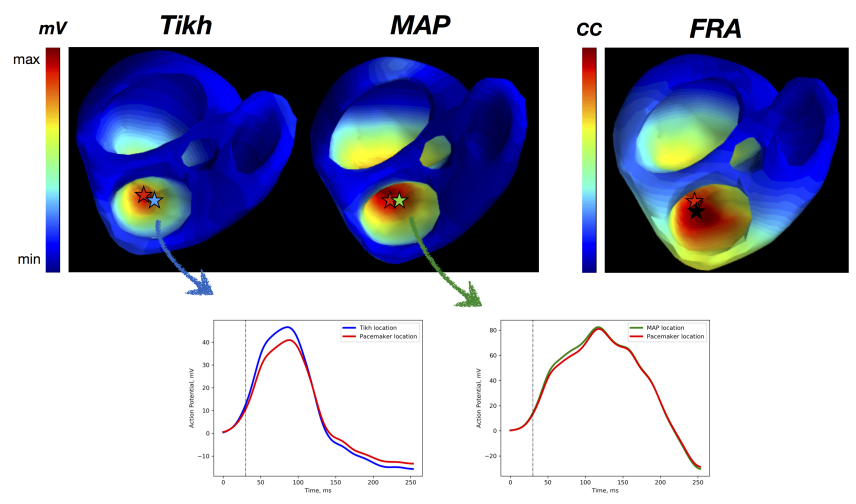

Figure 3: Exemplary reconstruction results for an endocardial RV pacing marked by a red star. For figure structure and color codes the reader is referred to Fig. 2. In contrast to the epicardial LV pacing, all ECGI reconstructions resulted in a significantly more compact area of the potential earliest excitation zone. Obtained TMPs associated with true and localized origins exhibited very similar temporal evolution similar to the LV case.

\section{Discussion and conclusions}

In this work, we evaluated ECGI performance in detection of the focal earliest activation zone in CRT patients. The three considered reconstruction methods covered the whole spectrum of inverse approaches. While Tikhonov regularization belongs to a classical data-driven module and FRA is a purely model-based routine, MAP with FRA sequences utilizes features of both.

For the LV paced sequences, Tikhonov and MAP showed superior activation onset localization, whereas all methods resulted in very similar accuracy for the RV cases. We relate this phenomenon to structural changes in CRT patients, who are characterized by a scar or slow conduction area in the LV. These substrate modification violates FRA modeling assumptions and deteriorates inverse solution quality. However, the FRA-generated activation sequences could be efficiently used as an approximation of the sources' covariance matrix. 

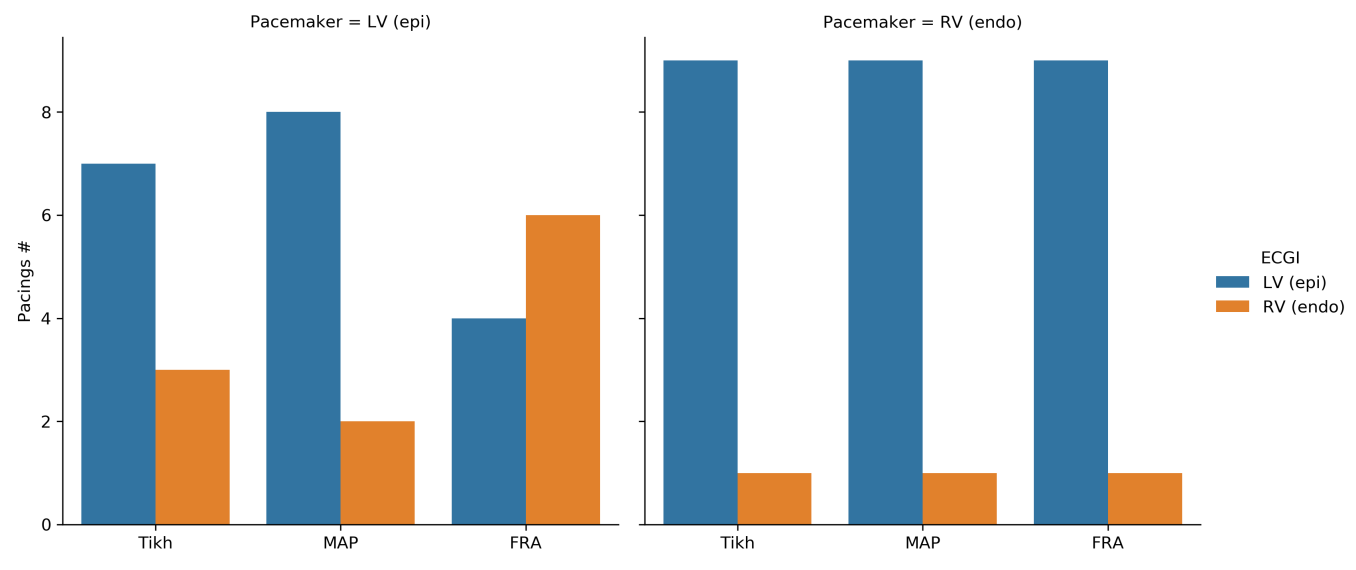

Figure 4: Number of ECGI detected epi- and endocardial activation onsets for the LV (left) and RV (right) caces. As all LV/RV pacings were delivered on the epi-/endocardial heart surface, an ideal noninvasive solution would result in blue bars/orange bars for the LV/RV cases, respectively.

\section{Acknowledgments}

The authors would like to thank the Department of Radiology in Almazov National Medical Research Center (Saint-Petersburg) for the strong commitment. Furthermore, we highly appreciate support from Dr. Andrey Kozlenok, the head of Department for Cardiovascular Physiology, in making this study possible.

\section{References}

[1] Bayley RH, Berry PM. The electrical field produced by the eccentric current dipole in the nonhomogeneous conductor. Amer Heart J 1962;63(6):808-820.

[2] McFEE R, Baule GM. Research in electrocardiography and magnetocardiography. IEEE Proc 1972;60(3):290-321.

[3] Barr RC, Spach MS. Inverse calculation of qrs-t epicardial potentials from body surface potential distributions for normal and ectopic beats in the intact dog. Circ Research 1978; 42(5):661-675.

[4] Rudy Y, Plonsey R, Liebman J. The effects of variations in conductivity and geometrical parameters on the electrocardiogram, using an eccentric spheres model. Circ Research 1979;44(1):104-111.

[5] Rudy Y, Burnes JE. Noninvasive electrocardiographic imaging. Ann Noninvasive Electrocardiol 1999;4(3):340359.

[6] Cluitmans M, Brooks DH, MacLeod R, Dssel O, Guillem MS, van Dam PM, Svehlikova J, He B, Sapp J, Wang L, Bear L. Validation and opportunities of electrocardiographic imaging: From technical achievements to clinical applications. Front Physiol 2018;9:1305. ISSN 1664-042X.

[7] He B, Li G, Zhang X. Noninvasive imaging of cardiac transmembrane potentials within three-dimensional myocardium by means of a realistic geometry anisotropic heart model. IEEE Trans Biomed Eng 2003;50(10):1190-1202.

[8] Skipa O. Linear inverse problem of electrocardiogra- phy: epicardial potentials and transmembrane voltages. Helmesverl., 2004.

[9] Geselowitz DB. Description of cardiac sources in anisotropic cardiac muscle: application of bidomain model. J Electrocardiol 1992;25:65-67.

[10] Van Oosterom A. Genesis of the $t$ wave as based on an equivalent surface source model. J Electrocardiol 2001; 34(4):217-227.

[11] van Oosterom A, Jacquemet V. A parameterized description of transmembrane potentials used in forward and inverse procedures. Folia Cardiologica 2005;12(suppl D):111.

[12] Van Dam PM, Oostendorp TF, Linnenbank AC, Van Oosterom A. Non-invasive imaging of cardiac activation and recovery. Ann Biomed Eng 2009;37(9):1739-1756.

[13] Gordon JP, Laks M, Boyle NG, van Dam PM. Advantages of the cardiac isochrone positioning system for localization of pves to the endocardium, epicardium, and midmyocardium from a 12 lead ecg, 2014.

[14] Erem B, Coll-Font J, Orellana RM, St'ovicek P, Brooks DH. Using transmural regularization and dynamic modeling for noninvasive cardiac potential imaging of endocardial pacing with imprecise thoracic geometry. IEEE Trans Medi Imag 2014;33(3):726-738.

[15] van Oosterom A. The use of the spatial covariance in computing pericardial potentials. IEEE Trans Biomed Eng 1999;46(7):778-787.

[16] Schulze WH. ECG imaging of ventricular activity in clinical applications, volume 22. KIT Scientific Publishing, 2015.

Address for correspondence:

Danila Potyagaylo

EP Solutions SA,

Avenue des Sciences 13, 1400 Yverdon-les-Bains, Switzerland contact@ep-solutions.ch 